\title{
De novo MECP2 duplication in two females with random X-inactivation and moderate mental retardation
}

\author{
Ute Grasshoff ${ }^{\star}, 1$, Michael Bonin ${ }^{1}$, Ina Goehring ${ }^{2}$, Arif Ekici², Andreas Dufke ${ }^{1}$, Kirsten Cremer ${ }^{3}$, \\ Nicholas Wagner ${ }^{3}$, Eva Rossier ${ }^{1}$, Anna Jauch ${ }^{4}$, Michael Walter ${ }^{1}$, Claudia Bauer ${ }^{1}$, Peter Bauer ${ }^{1}$, Karl Horber ${ }^{5}$, \\ Stefanie Beck-Woedl ${ }^{1}$ and Dagmar Wieczorek ${ }^{3}$
}

Xq28 duplications including MECP2 are a well-known cause of severe mental retardation in males with seizures, muscular hypotonia, progressive spasticity, poor speech and recurrent infections that often lead to early death. Female carriers usually show a normal intellectual performance due to skewed $\mathrm{X}$-inactivation $(\mathrm{XCl})$. We report on two female patients with a de novo MECP2 duplication associated with moderate mental retardation. In both patients, the de novo duplication occurred on the paternal allele, and both patients show a random $\mathrm{XCI}$, which can be assumed as the triggering factor for the phenotype. Furthermore, we describe the phenotype that might be restricted to unspecific mild-to -moderate mental retardation with neurological features in early adulthood.

European Journal of Human Genetics (2011) 19, 507-512; doi:10.1038/ejhg.2010.226; published online 16 February 2011

Keywords: Xq28 duplication; MECP2; X-inactivation; mental retardation in females

\section{INTRODUCTION}

The X-linked Rett syndrome (OMIM \#312750) has been known for a long time as a mental retardation syndrome, with its first clinical description in 1966/1977, and the identification of its underlying cause with loss-of-function mutations of the MECP2 gene in $1999.1^{1-3}$ The Rett syndrome is almost always sporadic because of de novo mutations and is seen almost exclusively in girls. Typical findings of classic Rett syndrome are a regression of motor skills, communicative abilities and especially a loss of purposeful hand movements, accompanied by secondary microcephaly after a period of (near to) normal development. The few affected males with deleterious MECP2 mutations usually present with clinical features consisting of fatal seizures and severe mental retardation including microcephaly that often leads to death during the initial years. The genetically related Lubs syndrome (OMIM \#300260) was first described in 1999, and etiologically resolved in 2005 as the MECP2 duplication syndrome. ${ }^{4-6}$ Xq28 duplications of different sizes spanning the MECP2 gene have been identified to date as a common cause of mental retardation in males. In familial cases with $\mathrm{X}$-linked pedigrees, the asymptomatic female carriers show a significant skewing of X-inactivation (XCI) ${ }^{7-9}$ Besides mental retardation, the main features of the affected boys are muscular hypotonia, progressive spasticity, seizures, poor speech and recurrent infections. The reported duplications vary from 0.1 to $2.6 \mathrm{Mb}$ with the minimal critical region containing the IRAK and MECP2 genes. ${ }^{7}$ In familial cases, carrier females might suffer from endocrinological abnormalities, autoimmune diseases, as well as psychiatric disorders. ${ }^{10}$ A phenotype with mental retardation has been reported only two times. ${ }^{11,12}$
We report on two unrelated female patients with a de novo MECP2 duplication and a random XCI, revealing a moderate but unspecific mental retardation in childhood and the development of neurological features in the second decade of life.

\section{MATERIALS AND METHODS}

\section{Patients}

Both patients were examined for diagnostic evaluation because of mental retardation. Karyotype analysis was performed for each patient on metaphase chromosomes from cultured blood lymphocytes using Giemsa banding at 550 bands resolution with normal results. The parents of patient 1 also showed normal karyotypes. Informed consent for the investigation was obtained from both families.

\section{SNP microarray}

Genomic DNA was isolated from peripheral blood leukocytes using routine procedures. In both samples, we used the Affymetrix Genome-Wide Human SNP Array 6.0 (Affymetrix, Santa Clara, CA, USA) with an average distance of $1.3 \mathrm{~kb}$ between neighboring probes. Samples were processed according to the manufacturer's instructions. Genotypes were called with Affymetrix Genotyping Console Software v3 (GTC) using the Birdseed algorithm with a default calling threshold of 0.5 and a prior size of 10000 bases in a simultaneous analysis of the patient-parents trio. Interpretation was based on Human Genome Build 36 (NCBI).

\section{Multiplex ligation-dependent probe amplification (MLPA)}

In patient 2, MECP2 duplication was confirmed by multiplex ligationdependent probe amplification with the test kit P015D2 Human Probe Mix by MRC Holland, Amsterdam, The Netherlands (http://www.mlpa.com),

${ }^{1}$ Department of Medical Genetics, University of Tübingen, Tübingen, Germany; ${ }^{2}$ Institute for Human Genetics, University of Erlangen, Erlangen, Germany; ${ }^{3}$ Institute of Human Genetics, University Hospital Essen, University of Duisburg-Essen, Essen, Germany; ${ }^{4}$ Institute of Human Genetics, University of Heidelberg, Heidelberg, Germany; ${ }^{5}$ Clinics of Neurology, University of Tübingen, Tübingen, Germany

*Correspondence: Dr U Grasshoff, Department of Medical Genetics, University of Tübingen, Calwerstreet 7, Tübingen 72076, Germany. Tel: +49 70712972290 ;

Fax +49 707129 5171; E-mail: ute.grasshoff@med.uni-tuebingen.de

Received 2 June 2010; revised 17 November 2010; accepted 18 November 2010; published online 16 February 2011 
according to the manufacturer's instructions. The multiplex ligation-dependent probe amplification profiles were obtained using normal and pathological DNA samples as controls.

\section{Quantitative PCR (qPCR)}

In patient 2, the relative copy number of selected genomic regions was determined by quantitative PCR as described previously (data not shown).

\section{X-inactivation (XCI)}

The highly polymorphic (CAG)n motif within the human androgen receptor gene (HUMARA) was used to distinguish and compare the methylation activity of the $\mathrm{X}$ chromosomes in both patients as described previously. ${ }^{13}$

\section{Fluorescence in situ hybridization (FISH)}

To investigate the localization of the duplicated segment, FISH analyses were performed on metaphase spreads and interphase nuclei of both patients and the parents of patient 2. Using standard two-color FISH protocols, a BAC clone from the duplicated region Xq28 (RP11-218L14, kindly provided by Joris Vermeesch, Center for Human Genetics, Leuven, Belgium) was hybridized together with a whole or partial chromosome painting probe for chromosome X (wcpX or pcpXq).$^{14}$ Microscopic evaluation of 20 metaphase spreads and 100 nuclei from each patient and the parents of patient 2 was performed using a DM RXA epifluorescence microscope (Leica Microsystems, Bensheim, Germany) equipped with a Sensys CCD camera (Photometrics, Tucson, AZ, USA).

\section{Microsatellite analysis}

The parental origin of the duplication was determined by microsatellite analysis, and the signal intensities for specific probes were determined on the 6.0 array. Microsatellite analysis was performed with four di-nucleotide sequences within the region of interest. Motifs were selected using the UCSC (University of California) genome browser and repeat-spanning primers were designed with a $5^{\prime}$-fluorescence tag for the forward primer. PCR amplicons for trios were analyzed using capillary electrophoresis (Beckman Coulter CEQ8000; Beckman Coulter, Brea, CA, USA) and amplicon lengths were determined. Besides genotyping, we measured peak heights for all duplication carriers compared with normal controls in order to identify duplicated and nonduplicated alleles as described previously. ${ }^{15}$

The SNP-microarray data were evaluated considering the genotype and the corresponding probe signals within the duplicated region, thus providing a correlation of a specific genotype and the respective signal height. The origin of the duplication was identified by an allele-specific elevation of signal intensity in the patient in comparison with the parental genotypes.

\section{RESULTS}

All position coordinates given below are based on Human Genome Build 36.

\section{Case reports}

Patient 1. The girl was born to healthy, non-consanguineous parents of Turkish origin at 39 weeks gestation via forceps delivery with a birth weight of $2900 \mathrm{~g}(-1.0 \mathrm{SD})$, a length of $48 \mathrm{~cm}(-1.3 \mathrm{SD})$ and a head circumference of $34 \mathrm{~cm}(-0.3 \mathrm{SD})$. She had constipation from birth, for which she had to be treated with a laxative. During her initial years of life, she presented with a high frequency of respiratory infections. She learned to walk and spoke her first words at the age of 19 months. Her further psychomotor development was delayed. The parents noted autistic behavioral features in their daughter - for example, that she mostly played alone.

We examined patient 1 for diagnostic evaluation at the age of $6 \frac{2}{12}$ years and again at $7 \frac{6}{12}$ years. At second examination, her body measurements were still normal: her height was $128 \mathrm{~cm}(0.3 \mathrm{SD})$, her weight was $28.5 \mathrm{~kg}(0.9 \mathrm{SD})$ and her OFC was $52.5 \mathrm{~cm}(0.7 \mathrm{SD})$. She visited a school for the mentally retarded, and was not able to write or count. Her non-verbal IQ was 58. Her constipation improved, but sometimes a drug therapy was necessary. Adenoids were removed at the age of 5 years, but overall there was no history of recurrent or severe infectious diseases. She still displayed autistic behavior, spending most of her time engaged in role-playing games with her dolls. Her psychomotor development was slow, but the parents did not report regression, and no epilepsy or stereotypies were reported. She had no facial dysmorphism, but facial similarity to her father, who also presented with synophrys. Bilateral simian creases as well as a sacral hemangioma were noted. No other abnormalities were present.

Patient 2. Patient 2 was born at 40 weeks of gestation by spontaneous vaginal delivery as the third of four children of non-consanguineous Turkish parents following an uncomplicated pregnancy. Birth measurements were normal with a weight of $3650 \mathrm{~g}$ (0.4 SD), a length of $52 \mathrm{~cm}$ (mean) and an OFC of $35.5 \mathrm{~cm}(0.5 \mathrm{SD})$. Her three brothers were healthy. She achieved normal motor milestones. Speech development was delayed and started at 3 years. At the age of 5 years, the parents reported a stagnation of development, but no regression. She mostly played alone and revealed some autistic features. After a first year at regular school, she changed to a special school. She never learnt to read or write. At the age of 10 years, standardized non-verbal testing (SON) showed a developmental age of 6 years and a cognitive score of 55. On physical examination at the age of 10 years, her weight was $36.8 \mathrm{~kg}$ (BMI: 17.6, 50-70th centile), her height was $145 \mathrm{~cm}$ (0.9 SD) and her OFC was $54 \mathrm{~cm}(1.2 \mathrm{SD})$. She showed a primary enuresis until the age of 12 years, and, furthermore, a vesicourethral reflux $\mathrm{II}^{\circ}$ was diagnosed. The EEG at that age was irregular, with alpha-rhythm and abnormal beta waves. The brain MRI was normal.

At the age of 18 years, a spastic hypertonia of the legs appeared, which led to gait disturbances. EEG at the age of 20 years was physiological; MRI of the myelon revealed no pathological aspects. At 20 years, the patient's weight was $67 \mathrm{~kg}$ (BMI: 26.2, 90-97th centile), her height was $160 \mathrm{~cm}(-1 \mathrm{SD})$ and her OFC was $54 \mathrm{~cm}$ (mean). Patient 2 did not present with any dysmorphic features. Her course of puberty was described as normal and no endocrinological problems were reported.

\section{Detection of MECP2 duplications}

The de novo duplication of Xq28 material, including the MECP2 gene, was identified in both patients using array analyses. In patient 1 , a 266-kb duplication (chrX: $152892781-153158642,266 \mathrm{~kb}, 71$ array markers) containing seven reference sequence genes was detected (RefSeq, according to the genome browser of the UCSC). The proximal breakpoint was located within the TMEM187 gene, centromeric to the IRAK gene, and the telomeric breakpoint was located within the TEX28 gene (Figure 1). In addition, patient 1 showed a second de novo duplication of 2q37.3 material (chr2: 241714 171$241825938,112 \mathrm{~kb}, 71$ array markers), including the genes ANO7, HDLBP, PASK andPPP1R7. The genotype for patient 1 according to the International System for Human Cytogenetic Nomenclature 2009 (ISCN) is arr 2q37.3(241 714 171-241 825 938)×3 dn, Xq28(152 $892781-$ $153158642) \times 3 \mathrm{dn}$.

In patient 2, we found a duplication of chromosome Xq28 material (chrX: 152 925 421-153 403 154, $478 \mathrm{~kb}, 215$ array markers) containing 22 RefSeq genes (UCSC), with the proximal breakpoint centromeric to the IRAK gene and the distal breakpoint telomeric to the FAM $3 A$ gene (Figure 1). The nomenclature according to ISCN for patient 2 is arr Xq28(152 $925421-153403154) \times 3$ dn.

\section{Confirmation and delineation of $M E C P 2$ duplications}

In patients 1 and 2, the duplication in Xq28 was confirmed by interphase FISH based on the presentation of three hybridization signals 

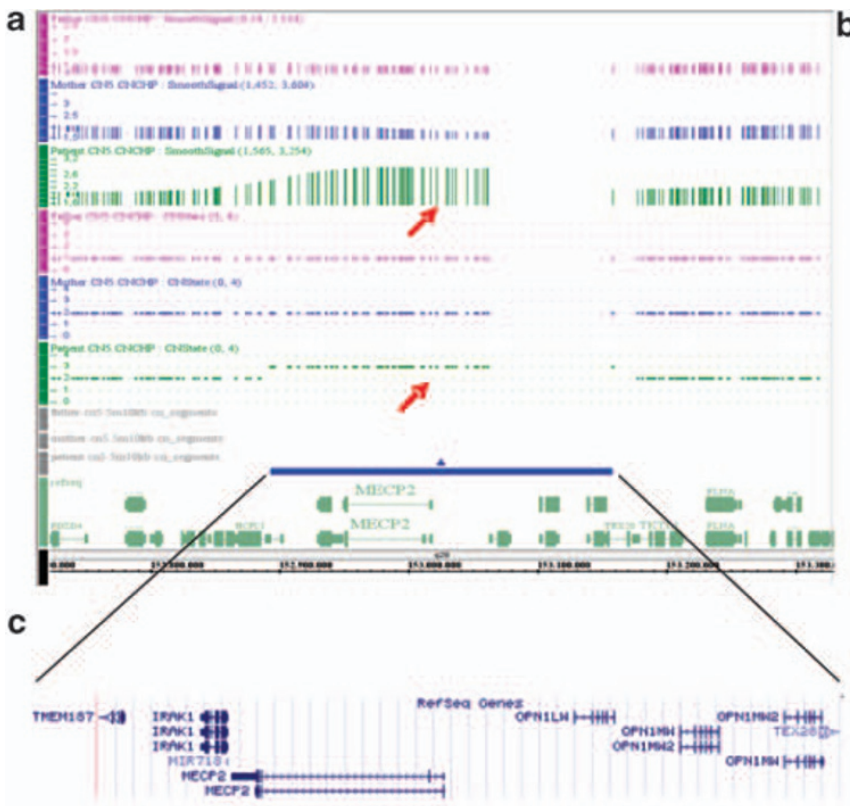

Patient 1

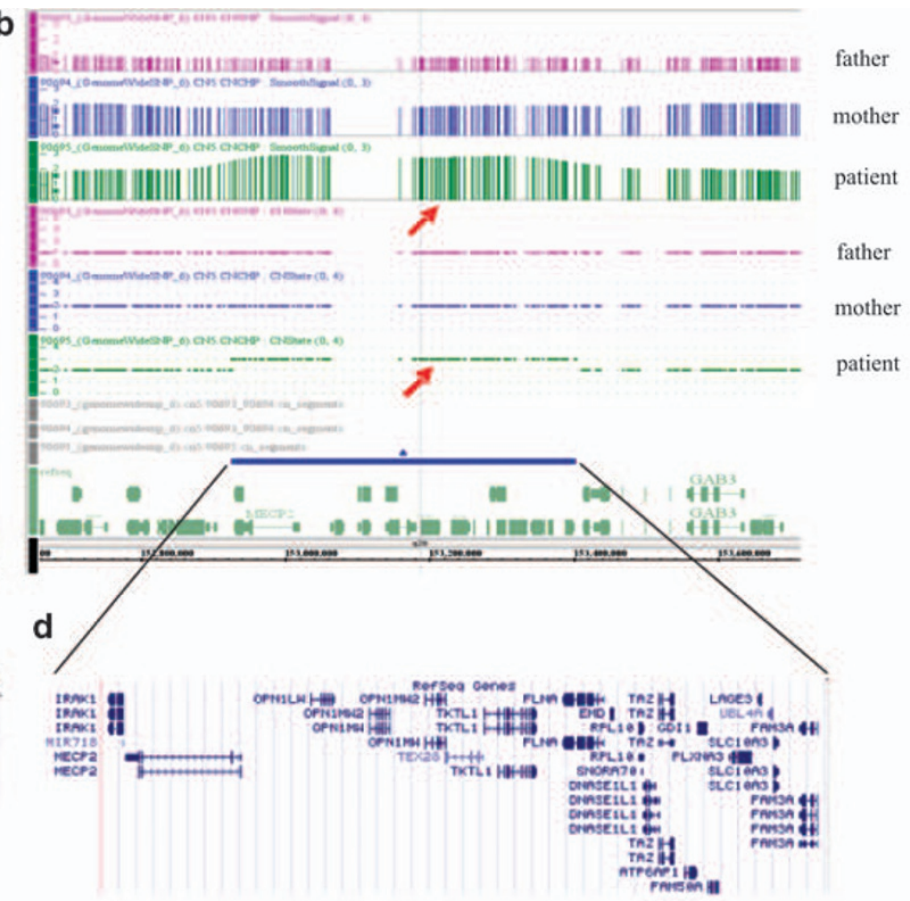

Patient 2

e

Asse mbly exceptions chromosome $X$

Asse mbly exceptions
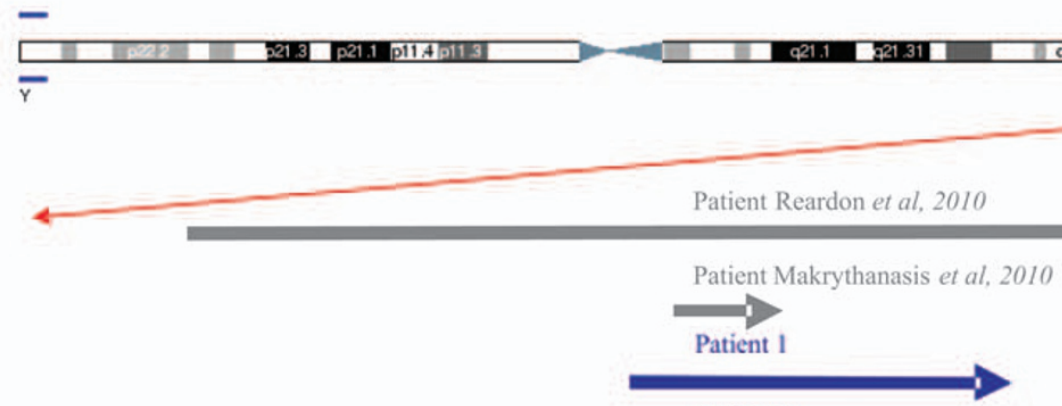

Patient 2

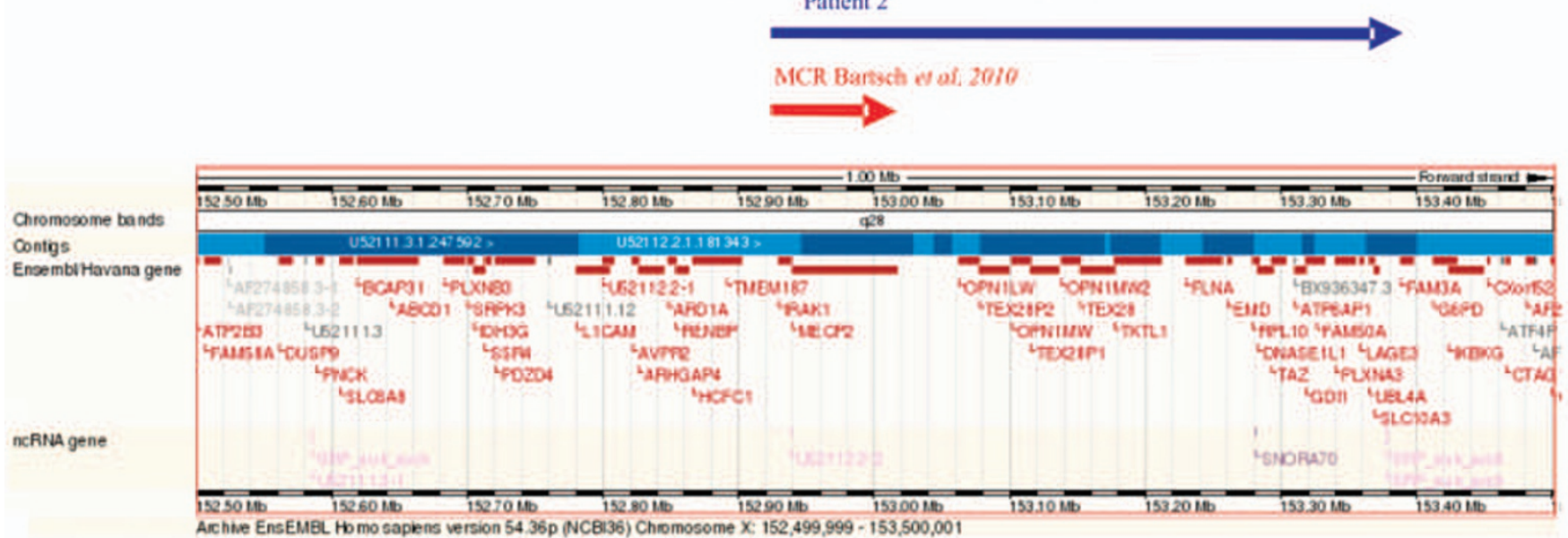

Figure 1 Illustration of the genomic aberration of the MECP2 region with the 6.0 Affymetrix SNP Array and schematic representation of the duplicated region. (a and $\mathbf{b}$ ) Image of the parents-patient trio analysis, showing the de novo occurrence of the duplication (red arrows) in both cases; (c and d) mapping of the duplicated regions according to UCSC; (e) schematic representation of part of the genomic region Xq28 with the location of the duplication of our two novel patients (blue arrows), the female patients of Reardon et a ${ }^{11}$ and Makrythanasis et a ${ }^{12}$ (gray arrows) as well as the minimal critical region (MCR) defined by Bartsch et $a P^{P}$ (red arrow). Gene content of the region is shown from the Ensembl genome browser version 54.36p (NCBI36).

for the BAC clone RP11-218L14 (Figure 2) A localization of the duplicated region on an autosome was excluded for both patients in metaphase spreads. In the parents of patient 2, FISH analyses for the
$\mathrm{Xq} 28$ BAC clone revealed only normal signals on the X chromosomes. Furthermore, duplications of the genes IRAK, MECP2 and GDI1 were confirmed in patient 2 according to the gene-specific probes from the 
multiple ligation-dependent probe amplification kit, whereas a normal signal was obtained for the probe against the proximal flanking IRAK gene. In patient 2, the duplication of $M E C P 2$ was confirmed by quantitative PCR (data not shown).

XCI studies showed a random XCI in both patients, with a status of 61:39 and 71:29, respectively (Figure 3). Microsatellite analysis revealed two alleles with different repeat lengths for the polymorphic marker in position chrX: $153050087-153050120(17 \times \mathrm{TG})$ in the father's and in the mother's DNA of patient 2. Semi-quantitative analysis indicates a paternal origin of the MECP2 duplication in patient 2 (data not shown). This marker and all other tested markers were not informative for patient 1 . The analysis of the SNP-microarray data regarding the correlation of genotype and signal height within the duplicated region indicates a paternal origin in both patients.
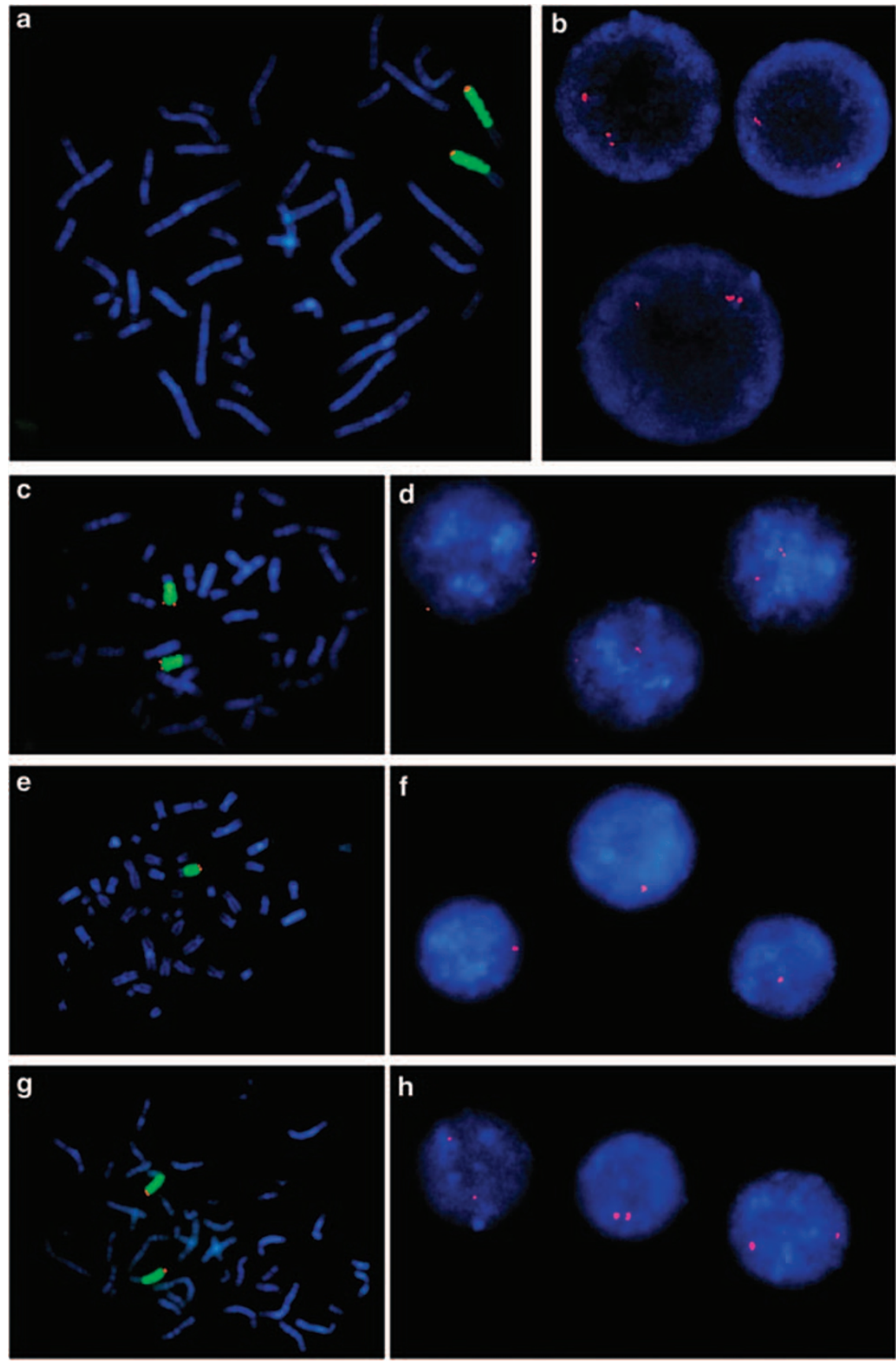

Figure 2 Metaphase and interphase FISH results. (a) Metaphase spread of patient 1 hybridized with the BAC clone RP11-218L14 localized in the duplicated chromosome region Xq28 (red) together with a partial chromosome painting probe for the long arm of the X chromosome (pcpXq, green). Signals are only present on both $\mathrm{X}$ chromosomes. (b) Interphase nuclei of the same patient, presenting a normal hybridization signal and a duplicated signal for the Xq28 BAC clone (red). (c) Metaphase spread of patient 2 after hybridization of the Xq28-BAC clone (RP11-218L14, red) together with a chromosomepainting probe for the $X$ chromosome (wcpX, green). Signals are only present on both $X$ chromosomes. (d) Interphase nuclei of patient 2 showing three hybridization signals, a normal signal and a duplicated signal for the Xq28 BAC clone (red). (e) Metaphase spread of the father of patient 2 presenting only one signal on the $\mathrm{X}$ chromosome and (f) a normal signal in the interphase nuclei with the Xq28 BAC clone. (g) Metaphase spread of the mother of patient 2 showing a specific hybridization signal on both $\mathbf{X}$ chromosomes and (h) two normal signals in the interphase nuclei. 
Patient 1

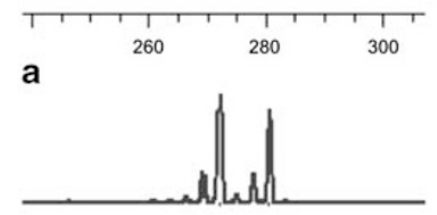

b
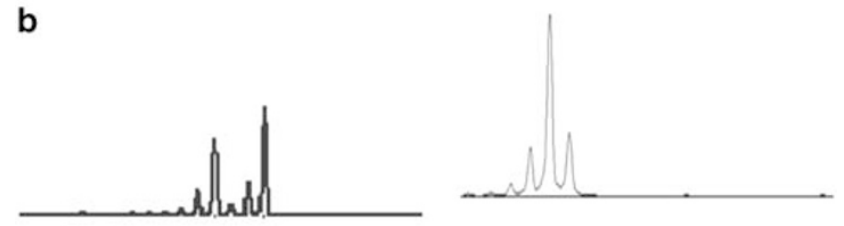

C
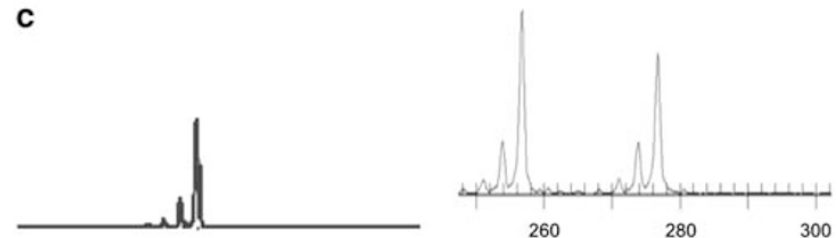

d
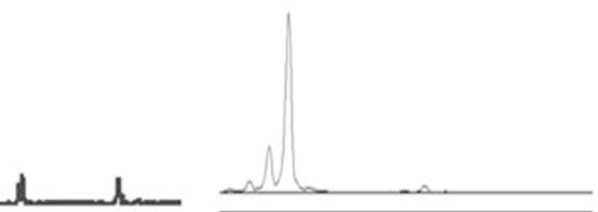

Figure $3 \mathrm{XCl}$ results. Results showing random $\mathrm{X}$ inactivation in both patients and proof of complete digestion with methylation-sensitive restriction endonuclease Hpall after PCR amplification of HUMARA (male control and female positive control, respectively). For patient 1 (performed on $A B I 3100$, Genotyper v3.7), trace (a) shows the undigested sample, trace (b) the digested fragments. The lower two traces show the male control (c) undigested and (d) after digestion. The $\mathrm{XCl}$ in patient 1 is 39:61. For patient 2 the upper two traces show the patient's sample (a) undigested and (b) digested (CEQ8000, Beckman Coulter), revealing a random $\mathrm{XCl}$ of 29:71. The lower traces show a female positive control with complete skewing of $\mathrm{X}$-inactivation in (c) undigested and (d) digested samples.

\section{DISCUSSION}

There were a considerable number of reports on mentally retarded boys with MECP2 duplications during the years following the first publication. ${ }^{6}$ However, to the best of our knowledge, only two mentally retarded females with an assured duplication have been reported. ${ }^{11,12}$ This underrepresentation of girls may have different reasons. An investigation bias favoring affected boys to be investigated for MECP2 duplications may have occurred, while girls were simply not investigated for this disorder. The fact that all females carrying the duplication were described as intellectually normal could have added to that effect. There is a recent report that considers certain clinical findings in those 'carrier mothers' as an affinity to endocrinological abnormalities (eg, hypothyroidism, menstrual irregularity and diabetes), autoimmune diseases, as well as psychiatric disorders (especially depression, anxiety and compulsion). ${ }^{10}$ Nevertheless, the intellectual performance of all investigated females in that study was in the normal range (full scale IQ 81-123). During the previous years, large cohorts of mentally retarded patients of both sexes were investigated with high-resolution arrays for submicroscopic genomic imbalances through different array technologies. These should have shown MECP2 duplications in females, and yet no further affected girls have been reported. ${ }^{16}$ This contradicts the investigation bias as an explanation for the rarity of those cases. We conclude that MECP2 duplications are a comparatively rare cause of mental retardation in females. This is confirmed by the identification of only two cases out of our cohort of 1000 unselected patients with mental retardation (incidence of 0.002).

In this study, we demonstrate two more female patients with an MECP2 duplication, and further delineate the aspect that, as Reardon et $a l^{11}$ first suggested, the random XCI may be causative for the phenotype. Most of the published carrier women who were informative for the XCI test presented a highly skewed XCI, with the duplication-bearing $\mathrm{X}$ chromosome being preferentially inactivated. ${ }^{10}$ One can reasonably assume that the random XCI is the crucial point leading to the mental retardation phenotype in these patients, whereas a highly skewed XCI with a preferential inactivation of the duplication-bearing X-chromosome is a protective factor. The impact of the XCI in our patients is further emphasized by the fact that a translocation of the duplicated segment to an autosome was excluded by FISH investigation. Another remarkable aspect of our patients, besides their retardation and the random $\mathrm{XCI}$, is that the MECP2 duplication occurred de novo in both cases. We propose that de novo occurrence of the MECP2 duplication, as in our cases, might have an effect on the XCI by preventing a protective 'mutation-induced' skewed XCI by a yet unrecognized mechanism. This would mean that there could be a different trigger for the methylation process from an inherited $M E C P 2$ duplication than from the one that occurred de novo. Furthermore, analysis of the microsatellites and the signal intensities of the microarrays for our patients showed that the de novo MECP2 duplication had been derived from the paternal $\mathrm{X}$ chromosome, which is a novelty in the literature.

For all but one female case reported so far, a duplication on the maternal X-chromosome can be assumed because of an X-linked pedigree. In this case, the duplication occurred de novo on an autosome (chromosome 10), thus completely escaping XCI. ${ }^{12}$

A post-zygotic mosaic occurrence of the duplication is a feasible explanation for a random XCI, and mosaicism cannot be excluded by any means. However, both patients showed no clinical signs of mosaicism, such as pigmental abnormalities of the skin or asymmetry, and the SNP-arrays gave no evidence for a mosaic state of the MECP2 duplication. This contradicts a high-level post-zygotic mosaicism as a cause for the random XCI in our cases.

It has to be mentioned that in patient 1 , a second de novo duplication was detected spanning $112 \mathrm{~kb}$ of chromosome $2 \mathrm{q} 37$ material with the RefSeq genes PASK, PPP1R7, ANO7 and HDLBP (UCSC:NCBI36/hg18). Only weak PASK (OMIM *607505) expression is found in human thymus and testis. PPP1R7 (OMIM ${ }^{\star} 602877$ ) is expressed in all human tissues including the brain. ANO7 (OMIM ${ }^{\star} 605096$ ) is expressed only in the prostate. The haploinsufficiency of two of the genes within this region has been associated to neuropsychiatric disorders $\left(H D L B P\right.$ and $\left.P A S K^{17}\right)$, but to our knowledge no phenotype has been demonstrated for a duplication of these genes. Thus, the significance of the $2 \mathrm{q} 37$ duplication remains unclear. An effect of the $2 \mathrm{q} 37$ duplication on the phenotype of the patient seems to be unlikely, but cannot be completely excluded. Regarding that, the phenotypic effect of a MECP2 duplication is already well documented, and patient 1 presents no clinical signs of a neuropsychiatric disorder 
Table 1 Clinical findings in female patients with MECP2 duplication and random $\mathrm{X}$-inactivation

\begin{tabular}{|c|c|c|c|c|}
\hline & Patient 1 & Patient 2 & Reardon et al ${ }^{12}$ & Makrythanasis et a/ ${ }^{13}$ \\
\hline Age at last examination (years) & $7 \frac{6}{12}$ & 20 & 12 & 18 \\
\hline MECP2 duplication & De novo & De novo & Maternal & De novo \\
\hline Mental retardation & Moderate & Moderate & Mild & Apparent, not specified \\
\hline Autistic features & + & + & n.r. & - \\
\hline Speech & Good & Good & Good & Simple \\
\hline Seizures & - & - & - & - \\
\hline Neurological symptoms & - & Leg spasticity & - & Poor coordination, eye movements \\
\hline Facial dysmorphism & - & - & - & - \\
\hline Constipation & + & - & n.r. & - \\
\hline Frequent infections in early childhood & + & - & n.r. & n.r. \\
\hline Bilateral simian creases & + & - & n.r. & - \\
\hline Sacral hemangioma & + & - & n.r. & - \\
\hline Vesicourethral reflux & n.r. & + & n.r. & - \\
\hline Endocrinological problems & n.r. & - & n.r. & + \\
\hline
\end{tabular}

Abbreviation: n.r., not reported.

exceeding the phenotype of patient 2; we feel it safe to assume that the phenotype is caused by the MECP2 duplication in both patients.

The typical symptoms of affected boys, such as seizures, poor speech development and recurrent severe infections, are not present in the females. The clinical signs in female patients with MECP2 duplication and random XCI consist of unspecific mild-to-moderate mental retardation, with autistic features, recurrent infections in early childhood, constipation and late-onset neurological features as variable symptoms. Facial dysmorphism was absent in all females (Table 1). ${ }^{18}$ This unspecific phenotype does not allow the diagnosis of females with a MECP2 duplication and random XCI on clinical grounds alone. Nevertheless, the stagnation of development at the age of 5 years in patient 2 , as well as the onset of spasticity in this patient at the age of 18 years, seems remarkable, especially regarding the progressive spasticity in males. This underlines the necessity to consider late-onset neurological features as a possible aspect in affected females.

In conclusion, we report on two female patients with a de novo MECP2 duplication and a mental retardation. Both patients show a random XCI, which can be postulated as the triggering factor for the phenotype. Furthermore, these are the first patients with a duplication derived from the paternal $\mathrm{X}$ chromosome and this report gives a description of the phenotype of affected females.

\section{CONFLICT OF INTEREST}

The authors declare no conflict of interest.

\section{ACKNOWLEDGEMENTS}

This study would not have been possible without the invaluable assistance of the patients and their families. We wish to thank the cooperating physicians for referral of the patients to our departments, especially G Dissemond,

L Kämmerling and V Horber. We also thank Bernhard Horsthemke, André Reis and Olaf Riess for continuous support, as well as Juliane Hoyer and Markus Zweier for their essential help. The Institutes for Human Genetics, Essen, Erlangen, Heidelberg and Tübingen, Germany, are members of the 'German Mental Retardation Network' (MRNET), which is funded by the German Federal Ministry of Education and Research (BMBF) as a part of the National Genome Research Network (http://www.ngfn.de/en/retardierung.html, project reference numbers 01GS08164, 01GS0816, 01GS08168, 01GS08162).
1 Rett A: Ueber ein eigenartiges hirnatrophisches Syndrom bei Hyperammoniamie in Kindesalter. Wien Med Wschr 1966; 116: 723-738.

2 Rett A: Cerebral atrophy associated with hyperammonaemia; in Vinken PJ, Bruyn GW (eds): Handbook of Clinical Neurology. Amsterdam: Elsevier, 1977, Vol 29, pp 305-329.

3 Amir RE, Van den Veyver IB, Wan M, Tran CQ, Francke U, Zoghbi HY: Rett syndrome is caused by mutations in X-linked MECP2, encoding methyl-CpG-binding protein 2. Nat Genet 1999; 23: 185-188.

4 Lubs H, Abidi F, Bier JAB et al: XLMR syndrome characterized by multiple respiratory infections, hypertelorism, severe CNS deterioration and early death localizes to distal Xq28. Am J Med Genet 1999; 85: 243-248.

5 Meins M, Lehmann J, Gerresheim F et al: Submicroscopic duplication in Xq28 causes increased expression of the MECP2 gene in a boy with severe mental retardation and features of Rett syndrome. (Letter). J Med Genet 2005; 42: e12.

6 Van Esch H, Bauters M, Ignatius J et al: Duplication of the MECP2 region is a frequent cause of severe mental retardation and progressive neurological symptoms in males. Am J Hum Genet 2005; 77: 442-453.

7 Bartsch O, Gebauer K, Lechno S et al: Four unrelated patients with Lubs X-linked mental retardation syndrome and different Xq28 duplications. Am J Med Genet A 2010; 152A: 305-312.

8 Friez MJ, Jones JR, Clarkson $\mathrm{K}$ et al: Recurrent infections, hypotonia, and mental retardation caused by duplication of MECP2 and adjacent region in Xq28. Pediatrics 2006; 118: e1687-e1695.

9 Vandewalle J, Van Esch H, Govaerts $\mathrm{K}$ et al: Dosage-dependent severity of the phenotype in patients with mental retardation due to a recurrent copy-number gain at Xq28 mediated by an unusual recombination. Am J Hum Genet 2009; 85: 809-822.

10 Ramocki MB, Peters SU, Tavyev YJ et al: Autism and other neuropsychiatric symptoms are prevalent in individuals with MeCP2 duplication syndrome. Ann Neurol 2009; 66: 771-782.

11 Reardon W, Donoghue V, Murphy AM et al: Progressive cerebellar degenerative changes in the severe mental retardation syndrome caused by duplication of MECP2 and adjacent loci on Xq28. Eur J Pediatr 2010; 169: 941-949.

12 Makrythanasis P, Moix I, Gimelli S et al: De novo duplication of MECP2 in a girl with mental retardation and no obvious dysmorphic features. Clin Genet 2010; 78: $175-180$.

13 Allen RC, Zoghbi HY, Moseley AB, Rosenblatt HM, Belmont JW: Methylation of Hpall and $\mathrm{Hhal}$ sites near the polymorphic CAG repeat in the human androgen-receptor gene correlates with X chromosome inactivation. Am J Hum Genet 1992; 51: 1229-1239.

14 Lichter $\mathrm{P}$, Cremer T: Chromosome analysis by non-isotopic in situ hybridization; in Roonie DE, Czepulkowski BH (eds): Human Cytogenetics - A Practical Approach. Oxford: Karger, 1992, pp 157-192.

15 Nguyen HP, Riess A, Krüger M et al: Mosaic Trisomy 21/Monosomy 21 in a living female infant. Cytogenet Genome Res 2009; 125: 26-32.

16 McMullan DJ, Bonin M, Hehir-Kwa JY et al: Molecular karyotyping of patients with unexplained mental retardation by SNP-arrays: a multicenter study. Hum Mutat 2009; 30: 1082-1092.

17 Felder B, Radlwimmer B, Benner A et al: FARP2, HDLBP and PASK are downregulated in a patient with autism and 2q37.3 deletion syndrome. Am J Med Genet A 2009; 149A: 952-959.

18 Clayton-Smith J, Walters S, Hobson E et al: Xq28 duplication presenting with intestinal and bladder dysfunction and a distinctive facial appearance. Eur J Hum Genet 2009; 17: 434-443. 